Network Working Group

Request for Comments: 4308

P. Hoffman

Category: Standards Track

VPN Consortium

December 2005

Cryptographic suites for IPsec

Status of This Memo

This document specifies an Internet standards track protocol for the Internet community, and requests discussion and suggestions for improvements. Please refer to the current edition of the "Internet Official Protocol Standards" (STD 1) for the standardization state and status of this protocol. Distribution of this memo is unlimited.

Copyright Notice

Copyright (C) The Internet Society (2005) •

Abstract

The IPsec, Internet Key Exchange (IKE), and IKEv2 protocols rely on security algorithms to provide privacy and authentication between the initiator and responder. There are many such algorithms available, and two IPsec systems cannot interoperate unless they are using the same algorithms. This document specifies optional suites of algorithms and attributes that can be used to simplify the administration of IPsec when used in manual keying mode, with IKEv1 or with IKEv2.

1. Introduction

This document is a companion to IPsec [RFC2401] and its two key exchange protocols, IKE [RFC2409] and IKEv2 [IKEv2]. Like most security protocols, IPsec, IKE, and IKEv2 allow users to chose which cryptographic algorithms they want to use to meet their security needs.

Implementation experience with IPsec in manual key mode and with IKE has shown that there are so many choices for typical system administrators to make that it is difficult to achieve interoperability without careful pre-agreement. Because of this, the IPsec Working Group agreed that there should be a small number of named suites that cover typical security policies. These suites may be presented in the administrative interface of the IPsec system. These suites, often called "UI suites" ("user interface suites"), are optional and do not prevent implementers from allowing individual selection of the security algorithms. 
Although the UI suites listed here are optional to implement, this document is on the standards track because implementers who call particular suites by the names used here have to conform to the suites listed in this document. These suites should not be considered extensions to IPsec, IKE, and IKEv2, but instead administrative methods for describing sets of configurations.

The key words "MUST", "MUST NOT", "SHOULD", "SHOULD NOT", and "MAY" in this document are to be interpreted as described in [RFC2119].

\section{UI Suites}

This section lists optional, non-mandatory suites that may be presented to system administrators to ease the burden of choosing among the many options in IPsec systems. These suites cannot cover all of the options that an administrator needs to select. Instead, they give values for a subset of the options.

Note that these UI suites are simply collections of values for some options in IPsec. Use of UI suites does not change the IPsec, IKE, or IKEv2 protocols in any way. Specifically, the transform substructure in IKE and IKEV2 must be used to give the value for each specified option regardless of whether or not UI suites are used.

Implementations that use UI suites SHOULD also provide a management interface for specifying values for individual cryptographic options. That is, it is unlikely that UI suites are a complete solution for matching the security policies of many IPsec users, and therefore an interface that gives a more complete set of options should be used as well.

IPsec implementations that use these UI suites SHOULD use the suite names listed here. IPsec implementations SHOULD NOT use names different than those listed here for the suites that are described, and MUST NOT use the names listed here for suites that do not match these values. These requirements are necessary for interoperability.

Note that the suites listed here are for use of IPsec in virtual private networks. Other uses of IPsec will probably want to define their own suites and give them different names.

Additional suites can be defined by RFCs. The strings used to identify UI suites are registered by IANA. 


\subsection{Suite "VPN-A"}

This suite matches the commonly used corporate VPN security used in IKEv1 at the time of this document's publication.

IPsec:

Protocol

Encapsulating Security Payload (ESP) [RFC2406]

ESP encryption

TripleDES in CBC mode [RFC2451]

ESP integrity

HMAC-SHA1-96 [RFC2404]

IKE and IKEV2:

Encryption TripleDES in CBC mode [RFC2451]

Pseudo-random function HMAC-SHA1 [RFC2104]

Integrity HMAC-SHA1-96 [RFC2404]

Diffie-Hellman group 1024-bit Modular Exponential (MODP) [RFC2409]

Rekeying of Phase 2 (for IKE) or the CREATE_CHILD_SA (for IKEv2) MUST be supported by both parties in this suite. The initiator of this exchange MAY include a new Diffie-Hellman key; if it is included, it MUST be of type 1024-bit MODP. If the initiator of the exchange includes a Diffie-Hellman key, the responder MUST include a DiffieHellman key, and it MUST of type 1024-bit MODP.

\subsection{Suite "VPN-B"}

This suite is what many people expect the commonly used corporate VPN security that will be used within a few years of the time this document's publication.

IPsec:

Protocol

ESP encryption

ESP integrity

IKE and IKEV2:

Encryption

Pseudo-random function

Integrity

Diffie-Hellman group
ESP [RFC2406]

AES with 128-bit keys in $\mathrm{CBC}$ mode [AES-CBC]

$\mathrm{AES}-\mathrm{XCBC}-\mathrm{MAC}-96$ [AES-XCBC-MAC ]

AES with 128-bit keys in $\mathrm{CBC}$ mode [AES-CBC]

$\mathrm{AES}-\mathrm{XCBC}-\mathrm{PRF}-128 \quad[\mathrm{AES}-\mathrm{XCBC}-\mathrm{PRF}-128]$

$\mathrm{AES}-\mathrm{XCBC}-\mathrm{MAC}-96 \quad[\mathrm{AES}-\mathrm{XCBC}-\mathrm{MAC}]$

2048-bit MODP [RFC3526]

Rekeying of Phase 2 (for IKE) or the CREATE_CHILD_SA (for IKEv2) MUST be supported by both parties in this suite. The initiator of this exchange MAY include a new Diffie-Hellman key; if it is included, it MUST be of type 2048-bit MODP. If the initiator of the exchange includes a Diffie-Hellman key, the responder MUST include a DiffieHellman key, and it MUST of type 2048-bit MODP. 


\subsection{Lifetimes for IKEv1}

IKEv1 has two security parameters that do not appear in IKEv2, namely, the lifetime of the Phase 1 and Phase 2 security associations (SAs). Systems that use IKEv1 with either the VPN-A or VPN-B suites MUST use an SA lifetime of 86400 seconds ( 1 day) for Phase 1 and an SA lifetime of 28800 seconds ( 8 hours) for Phase 2 .

\section{Acknowledgements}

Much of the text and ideas in this document came from earlier versions of the IKEv2 document edited by Charlie Kaufman. Other text and ideas were contributed by other members of the IPsec Working Group.

4. Security Considerations

This document inherits all of the security considerations of the IPsec, IKE, and IKEV2 documents.

Some of the security options specified in these suites may be found in the future to have properties significantly weaker than those that were believed at the time this document was produced.

5. IANA Considerations

IANA has created and will maintain a registry called, "Cryptographic Suites for IKEV1, IKEV2, and IPsec". The registry consists of a text string and an RFC number that lists the associated transforms. New entries can be added to the registry only after RFC publication and approval by an expert designated by the IESG.

The initial values for the new registry are:

Identifier Defined in

VPN-A RFC 4308

VPN-B RFC 4308 
6. Normative References

[AES-CBC] Frankel, S., Glenn, R., and S. Kelly, "The AES-CBC Cipher Algorithm and Its Use with IPsec", RFC 3602, September 2003.

$[\mathrm{AES}-\mathrm{XCBC}-\mathrm{MAC}]$

Frankel, S. and H. Herbert, "The AES-XCBC-MAC-96 Algorithm and Its Use With IPsec", RFC 3566, September 2003 .

[AES-XCBC-PRF-128] Hoffman, P., "The AES-XCBC-PRF-128 Algorithm for the Internet Key Exchange Protocol (IKE)", RFC 3664, January 2004 .

[ IKEv2 ]

Kaufman, C., Ed., "Internet Key Exchange (IKEv2) Protocol", RFC 4306, December 2005.

[RFC2104]

[RFC2119]

[RFC2401]

[RFC2 404 ]

[RFC2406]

[RFC2409]

[RFC2 4 51]

[RFC3526]

Krawczyk, H., Bellare, M., and R. Canetti, "HMAC: Keyed-Hashing for Message Authentication", RFC 2104, February 1997.

Bradner, S., "Key words for use in RFCs to Indicate Requirement Levels", BCP 14, RFC 2119, March 1997.

Kent, S. and R. Atkinson, "Security Architecture for the Internet Protocol", RFC 2401, November 1998 .

Madson, C. and R. Glenn, "The Use of HMAC-SHA-1-96 within ESP and AH", RFC 2404, November 1998.

Kent, S. and R. Atkinson, "IP Encapsulating Security Payload (ESP)", RFC 2406, November 1998.

Harkins, D. and D. Carrel, "The Internet Key Exchange (IKE)", RFC 2409, November 1998.

Pereira, R. and R. Adams, "The ESP CBC-Mode Cipher Algorithms", RFC 2451, November 1998.

Kivinen, T. and M. Kojo, "More Modular Exponential (MODP) Diffie-Hellman groups for Internet Key Exchange (IKE)", RFC 3526, May 2003. 


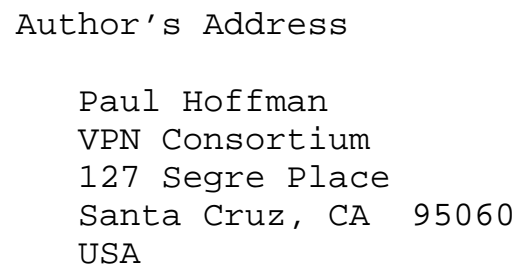

EMail: paul.hoffman@vpnc.org 
Full Copyright statement

Copyright (C) The Internet Society (2005).

This document is subject to the rights, licenses and restrictions contained in BCP 78, and except as set forth therein, the authors retain all their rights.

This document and the information contained herein are provided on an "AS IS" basis and THE CONTRIBUTOR, THE ORGANIZATION HE/SHE REPRESENTS OR IS SPONSORED BY (IF ANY), THE INTERNET SOCIETY AND THE INTERNET ENGINEERING TASK FORCE DISCLAIM ALL WARRANTIES, EXPRESS OR IMPLIED, INCLUDING BUT NOT LIMITED TO ANY WARRANTY THAT THE USE OF THE INFORMATION HEREIN WILL NOT INFRINGE ANY RIGHTS OR ANY IMPLIED WARRANTIES OF MERCHANTABILITY OR FITNESS FOR A PARTICULAR PURPOSE.

Intellectual Property

The IETF takes no position regarding the validity or scope of any Intellectual Property Rights or other rights that might be claimed to pertain to the implementation or use of the technology described in this document or the extent to which any license under such rights might or might not be available; nor does it represent that it has made any independent effort to identify any such rights. Information on the procedures with respect to rights in RFC documents can be found in BCP 78 and BCP 79 .

Copies of IPR disclosures made to the IETF Secretariat and any assurances of licenses to be made available, or the result of an attempt made to obtain a general license or permission for the use of such proprietary rights by implementers or users of this specification can be obtained from the IETF on-line IPR repository at http://www.ietf.org/ipr.

The IETF invites any interested party to bring to its attention any copyrights, patents or patent applications, or other proprietary rights that may cover technology that may be required to implement this standard. Please address the information to the IETF at ietfipreietf.org.

Acknowledgement

Funding for the RFC Editor function is currently provided by the Internet Society. 